\title{
Microwave glob top for space applications: A route to non hermiticity
}

\author{
$\underline{\text { P. MONFRAIX }}{ }^{(1)}$, L. ALBO $^{(1)}$, C. DREVO N ${ }^{(1)}$, \\ J.L. CAZAUX ${ }^{(1)}$, J.L. ROUX ${ }^{(2)}$
}

\begin{abstract}
(1) : ALCATEL SPACE INDUSTRIES, 26 avenue J.F. Champollion 31037 TO ULO USE, FRANCE
Phone: + 335343560 70, Fax: + 33534356947

E-mail : Philippe.Monfraix@space.alcatel.fr

(2) : CNES, 31000 TO ULO USE, France
\end{abstract}

\begin{abstract}
This paper presents the electrical design, measurement and reliability tests of an encapsulated MMIC using a dam \& fill technique. Materials, dispensing techniques, RF design are evaluated. Test samples have been designed and manufactured. Electrical results show a slight decay in frequency of the electrical characteristics (about $300 \mathrm{MHz}$ ) but the component still offers good performances in the relevant bandwidth for receivers.
\end{abstract}

\section{Introduction}

Current encapsulation techniques used for GaAs MMIC in space microwave equipment are based on metallic or ceramic hermetic packages. To provide the level of hermiticity currently needed, these solutions present some limits in term of integration and could be expensive. Glob top techniques, derived from low frequency applications, which consist of dispensing an organic material as an encapsulant on a bare die can offer size reduction eliminating the package and induce cost reduction as well.

Even if there is no perfect hermiticity in reference to MIL Std, several studies show there is no degradation induced by the glob top on GaAs MMIC with successful environmental tests such thermal cycling, $85^{\circ} \mathrm{C} / 85 \%$ residual humidity, ... Then this trend to move from hermiticity to a reliability without full hermiticity is a major evolution for space packaging. These great improvements are based on effort made from one part on the passivation of GaAs MMIC and another part on encapsulant such as the reduction of the coefficient of thermal expansion (from $60 \mathrm{ppm} /{ }^{\circ} \mathrm{C}$ in the 60 's down to a current value of $15 \mathrm{ppm} /{ }^{\circ} \mathrm{C}$ ) or the reduction of the $\mathrm{Cl}^{-}$ionic impurities by a factor 10.

\section{Materials}

Encapsulation materials used for glob top are organic one and have to fill lots of criterions : dielectric constant and dielectric losses stable with frequency and temperature, low ionic contaminants, a total mass loss below $1 \%$ and collected volatile condensable materials below $0.1 \%$. More than 20 epoxy, silicon and urethane based materials were investigated with characteristics in agreement with space requirements. For example, in the major samples evaluated, the ionic contaminants ratio has improved by a factor 50 to 100 from the last ten years and are now compatible to space requirements. These materials have 
also been electrically characterised in the $\mathrm{Ku}$ band, showing a dielectric constant around 3.5 and losses between $2.10^{-3}$ and $7.10^{-3}$.

\section{Dispensing techniques}

There are two solutions for dispensing techniques on a bare die (cf. Figure 2) : either do a glob top using one thixotropic material material (but the tolerance of the footprint on the substrate is quite high), either do a dam $\&$ fill (first, dispensing of a high viscosity encapsulant for a good accuracy of the position on the "organic wall", then filling of the cavity with a low viscosity material). ALCATEL has made the choice of a dam \& fill dispensing method. The conditions and methods needed for an accurate dispensing on microwave components have also been studied : the choice of dedicated valves, heating the substrate/the needle, the vertical position of the needle, ...

\section{RF design}

The encapsulated material has a great impact on the impedance of a RF line, mainly when it is a low dielectric constant substrate. For a microstrip design, the characteristic impedance of a line reduces by 3-4 $\Omega$ for a high dielectric substrate (typically alumina) and

6-7 $\Omega$ for a low dielectric substrate (typically Duroid $^{\circledR}$ ). For a coplanar design, the characteristic impedance of a line reduces by $4-5 \Omega$ for a high dielectric substrate and by $10-11 \Omega$ for a low dielectric substrate. Between coplanar and microstrip, the reason is explained through the field repartition. For high and low dielectric, the reason is the resin/substrate dielectric ratio, well balanced with low dielectric substrate. The width of an encapsulated line has then to be reduced. Simulations have showed that a good impedance controlled transmission line can be achieved through a tapered transition at the air/encapsulant interface. The length of the taper is a trade off between the electrical performances (as short as possible) and the dispensing mechanical tolerance.

\section{Electrical characterisation}

Samples (cf. Figure 2) have been manufactured. On wafer and glob topped MMIC electrical characterisations are presented on Figure 3. Due to an accurate dispensing method through dam $\&$ fill technique, we obtained a very good dimensional and electrical reproducibility on the different samples.

The blue curve represents the [S] parameters of the MMIC measured on wafer, whereas the black curve represents the [S] parameters of the MMIC packaged and glob topped. We can observe a slight decay in frequency of the electrical characteristics (about $300 \mathrm{MHz})$ but the component the component still offers good performances in the relevant bandwidth for receivers.

These samples have also been characterised with some environmental tests (thermal cycling, vapor phase, ...). Cross sections, specially at the encapsulant/MMIC interface, shows there is no impact on the air bridge located on the die.

\section{Condusion \& perspectives}

We presented in this paper a non hermetic solution to encapsulate microwave components for space applications. Materials, dispensing techniques, RF design transitions have been evaluated and proposed. Test vehicles have been manufactured and assembled for electrical validation. We can notice a slight decay in frequency 
of the electrical characteristics (about $300 \mathrm{MHz}$ ) but the component the component still offers good performances in the relevant bandwidth for receivers. Further improvements/studies could be proposed:

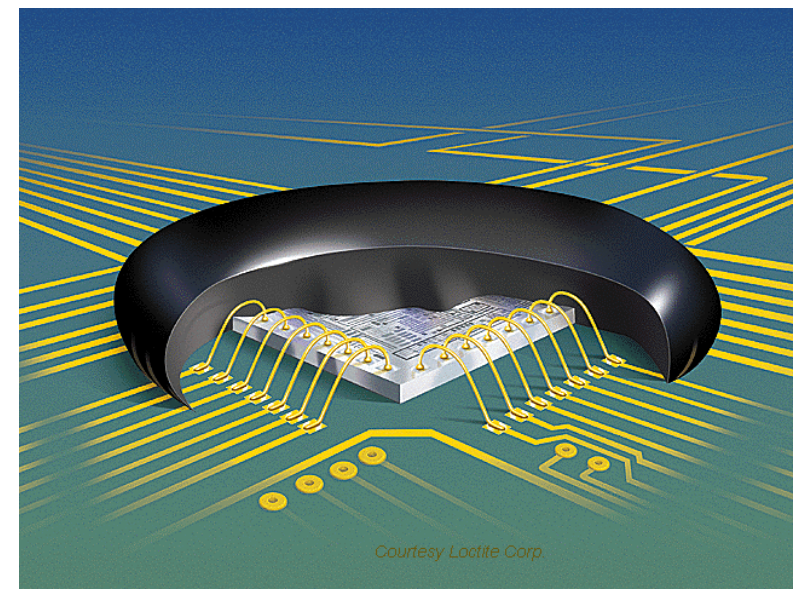

glob top electrical characterisation of this dispensing technique at Ka band,

evaluate the impact of environmental stresses as pressure cooker, high accelerated stress test, ... on this packaging.

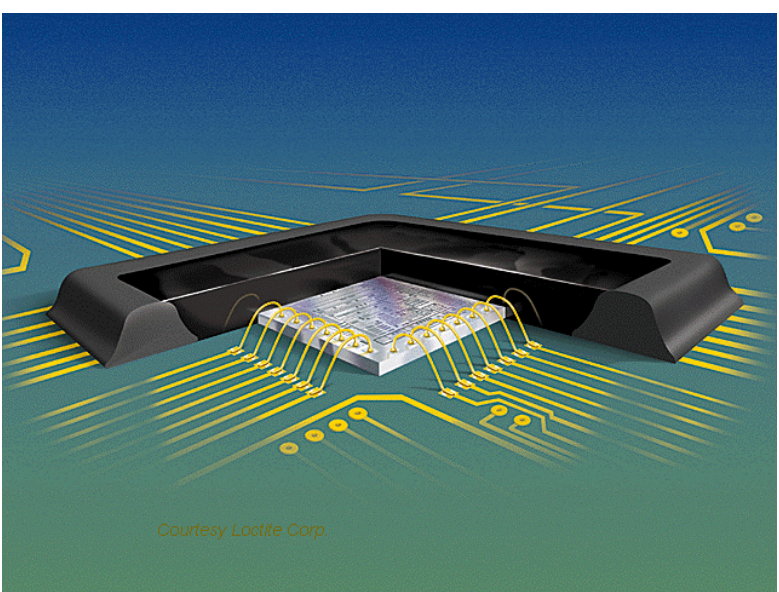

dam $\&$ fill

Figure 1 : Dispensing techniques.

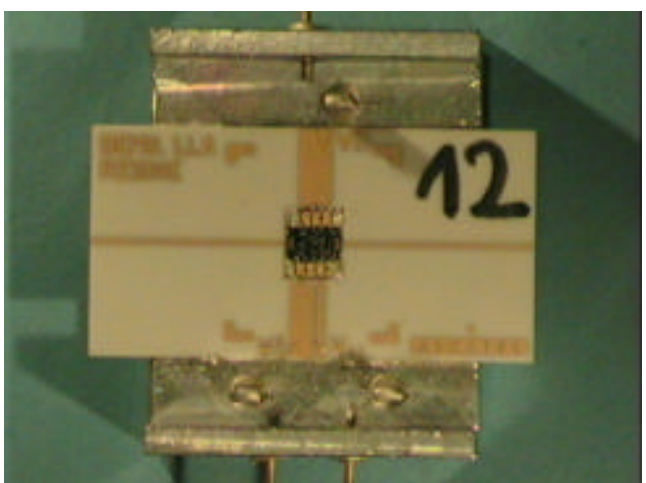

MMIC without glob top

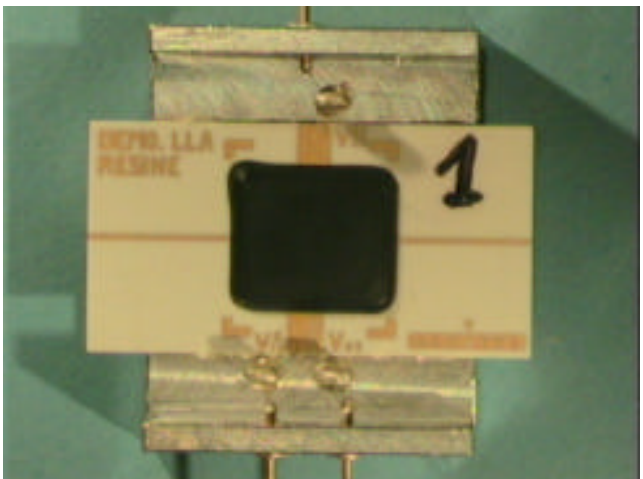

MMIC with glob top

Figure 2 : GaAs MMIC samples without and with glob top. 


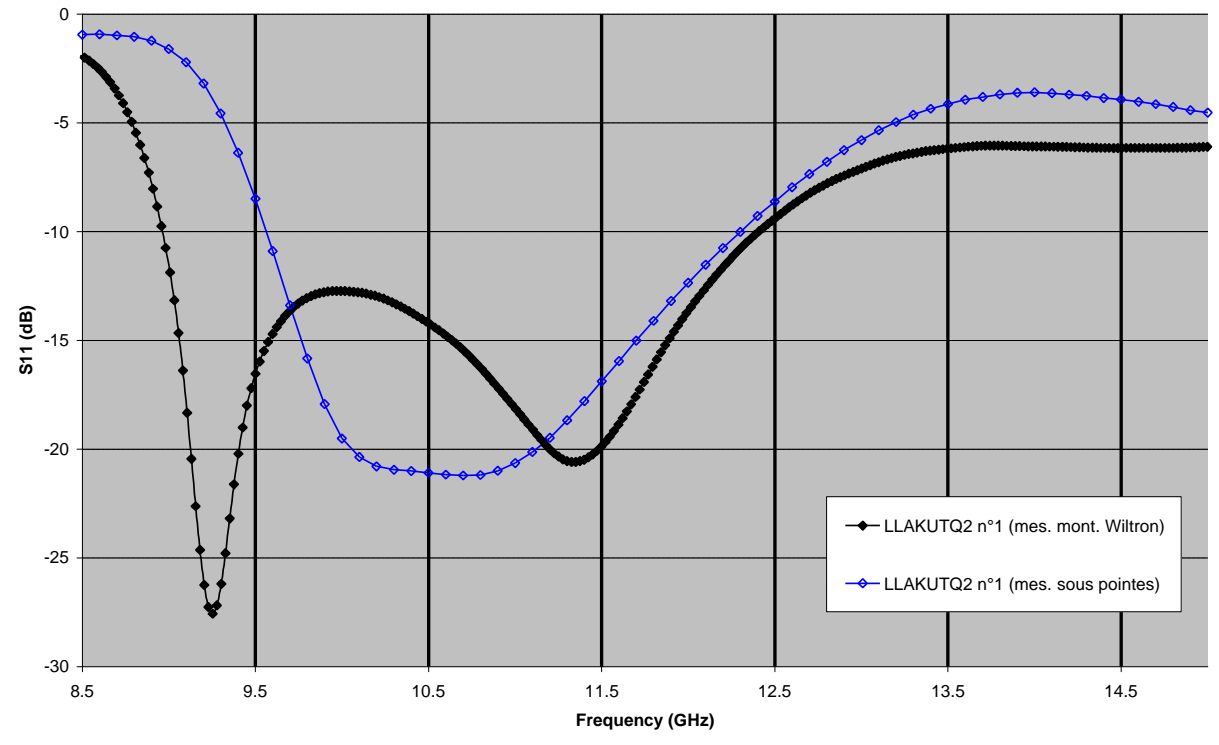

Return loss

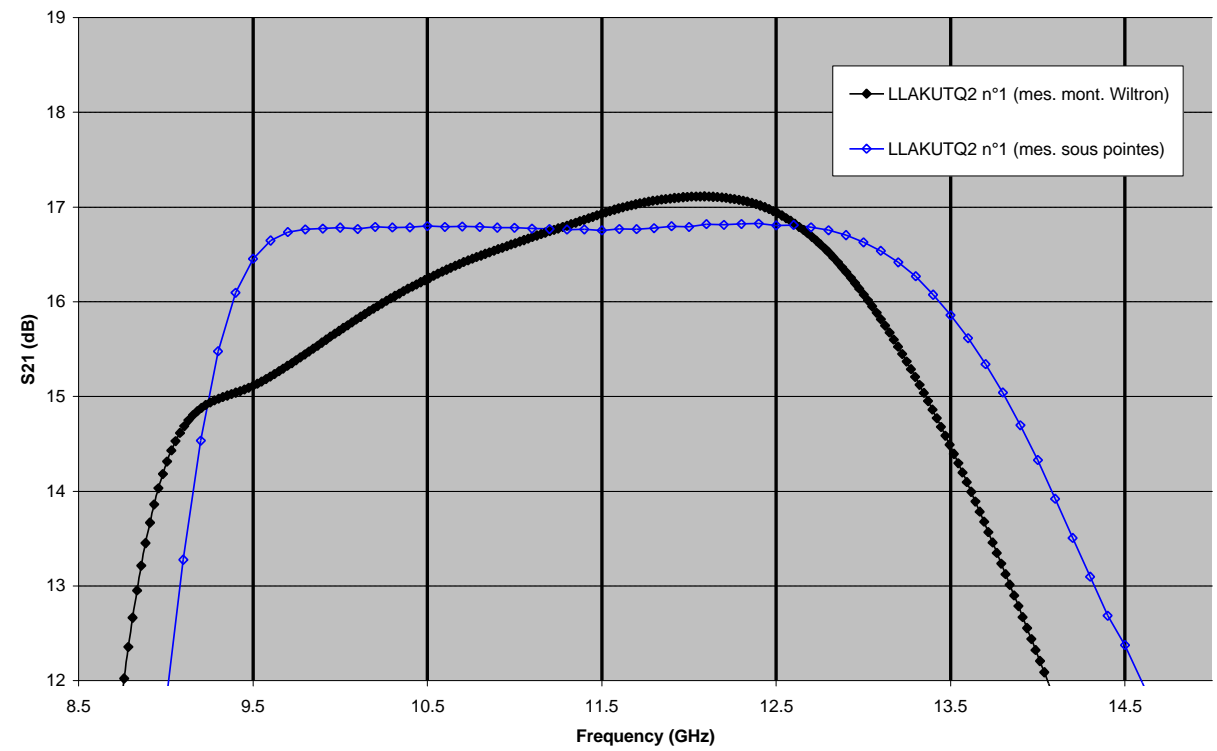

Gain

Figure 3 : GaAs MMIC electrical characteristics ([S] parameters) without (blue) and with (black) glob top. 